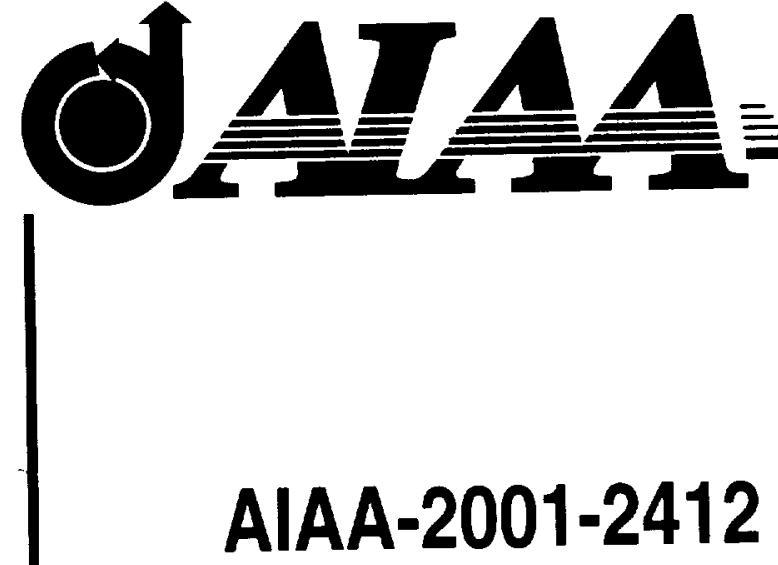 \\ A Boundary Condition for Simulation of Flow Over Porous Surfaces
}

\author{
N. Frink, D. Bonhaus, V. Vatsa, S. Bauer \\ NASA Langley Research Center \\ Hampton, VA
}

A. Tinetti

Virginia Polytechnic Institute and State Univ./VCES Hampton, VA

\section{9th Applied Aerodynamics Conference June 11-14, 2001/Anaheim, California}

For permission to copy or republish, contact the American Institute of Aeronautics and Astronautics 370 L'Enfant Promenade, S.W., Washington, D.C. 20024 


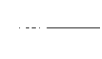




\title{
A BOUNDARY CONDITION FOR SIMULATION OF FLOW OVER POROUS SURFACES
}

\author{
Neal T. Frink*, Daryl L. Bonhaus, Veer N. Vatsa ${ }^{* *}$, Steven X. S. Bauer ${ }^{*}$ \\ NASA Langley Research Center \\ Hampton, Virginia, 23681
}

Ana F. Tinetti

Virginia Polytechnic Institute and State University / VCES

Hampton, Virginia 23681

\begin{abstract}
A new boundary condition is presented for simulating the flow over passively porous surfaces. The model builds on the prior work of R.H. Bush to eliminate the need for constructing grid within an underlying plenum, thereby simplifying the numerical modeling of passively porous flow control systems and reducing computation cost. Code experts for two structured-grid flow solvers, TLNS3D and CFL3D. and one unstructured solver. USM.3Dns, collaborated with an experimental porosity expert to develop the model and implement it into their respective codes. Results presented for the three codes on a slender forebody with circumferential porosity and a wing with leading-edge porosity demonstrate a good agreement with experimental data and a remarkable ability to predict the aggregate aerodinamic effects of surface porosity with a simple boundary condition.
\end{abstract}

\section{INTRODUCTION}

Passive Porosity Technology (PassPorT) [1] is an enabling flow alteration concept that can potentially resolve many aerodynamic problems. Its underlying principle is illustrated in the upper sketch of Fig. 1 that depicts a porous skin positioned over a closed cavity/plenum region. Local pressure differences within flow over the outer surface "communicate" through the plenum in concert with small amounts of mass transfer in and out of the porous surface to alter its effective aerodynamic shape. For a properly designed system. the hole size is small with respect to the boundary layer thickness and is less than or equal to the skin thickness, and the flow velocity into and out of the plenum is low. PassPorT was originally applied to transonic airfoils to reduce the normal shock strength and thus, eliminate shock-

* Senior Rescarch Engineer. Associate Fellow AIAA

** Senior Research Scientist. Member. AlAA

* Currently with Symantec, Inc.

+ Graduate student, Student Member AIAA

Conyright (1) $2(00)$ by the American Institule of Aeronatitics and Astronautics. Inc. No copyright is asserted in the United States under Titlo 17. U.S. Code. The U.S Government has a royalty-free license to exercise all rights under the copyright claimed herein for Governmental purposes All other rights are reserved hy the copyright owner
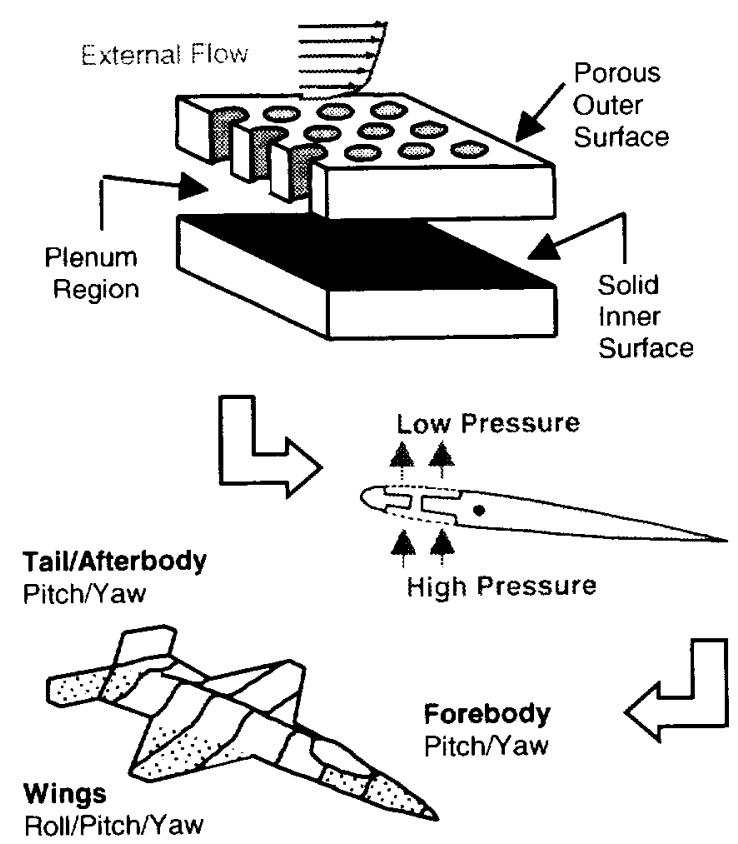

Figure 1 - The PassPorT concept and application as conformal control effector on military aircraft. 
induced separation and lower the drag levels [2-7].

More recently the concept was applied to a supersonic delta wing to reduce the crosstlow, shockinduced separation $[8]$. During this study, it was discovered that connecting the plenum to high and low pressure regions on the wing. as conveyed in the middle sketch in Fig. 1, allowed for localized "lift dumping" thus, providing roll and yaw control. Subsequently, many applications were envisioned such as conformal control effectors on military aircraft as illustrated on the lower half of Fig. 1. The potential for broad application of this technology to aerodynamic concepts provided enough incentive to fund the fabrication of a number of porous models.

A family of four Tangent-Ogive forebodies was built (two. 2.5 Caliber and two, 5.0-Caliber, each slenderness ratio consisted of one solid and one with $22 \%$ porosity surface). These models were lested in the NASA LaRC 7 - by 10-Foot High Speed Wind Tunnel, 14- by 22-Foot Subsonic Wind Tunnel, and Unitary Plan Wind Tunnel. In addition, the 5.0Caliber porous model was tested in the McDonnell Douglas Shear Flow Facility. The results from these tests $[9.10]$ showed that passive porous systems could be used to eliminate asymmetric loading conditions that occur at high angles of attack on axisymmetric slender bodies even at zero sideslip angle. This side force is a result of asymmetric pressure loading caused by differences in the location of the separation-induced vortex on either side of the forebody. This phenomenon has been documented extensively [1114|. Numerous studies have been conducted to develop devices that eliminate or minimize the asymmetric behavior of forebodies [15-18]. These "fixes" typically involve reshaping the nose or adding devices. such as strakes, to the existing geometry that add weight and are beneficial for only a limited range of conditions. In references 9 and 10 , a passive porous system was investigated to alleviate the asymmetric loading on the forebody. These tests also showed that pitch and yaw control could be attained hy opening or closing the porcus surface on one side or the other on the forebodies.

A zero-sweep, porous, $G A(W)-1$ [General Aviation (Whitcomb) - 1] [19] wing model was built and tested in the NASA LaRC 14- by 22-Fool Subsonic Wind Tunnel. All surfaces on the model were constructed with porosity that could be closed by covering the holes with lape. Through selective opening and closing of porous regions, the locations on the wing that induced the most pitch, yaw, and roll control were identified. as well as, the locations responsible for either increased performance (i.e., increased $L / \mathrm{D}_{\max }$ and angle of attack that $\mathrm{L} / \mathrm{D}_{\max }$ occurs) or increased drag. This model was later reskinned and modified to include actuators inside the plenums to open and close the porous surfaces. The results showed that no pressure lag occurred. The recorded forces responded as quickly as the porous surface could be actuated indicating no appreciable lag due to pressure equalization.

With the success achieved in the wind-tunnel tests, a series of research efforts were conducted to develop CFD models to represent a passive porous system. The first studies utilized Darcy's Law [20]. These had limited success and were fairly accurate as long as the coefficient required in the equation was chosen correctly. Later attempts with modified versions of Darcy"s Law and utilizing some techniques used by researchers to determine oxygen transport through capillary walls were slightly more successful [21].

The work presented in this paper builds on a methodology first developed by McDonnell Douglas to simulate normal flow through a screen positioned at a zonal-grid interface boundary [22]. The primary contribution of this work is to reformulate that approach into a new boundary condition for simulating the flow over porous aerodynamic surfaces. This eliminates the need for constructing grid within an underlying plenum. thereby simplifying the numerical modeling of passively porous flow control systems and reducing computation cost. Code experts for two structured-grid flow solvers. TLNS3D and CFL.3D. and one unstructured solver. USM3Dns. collaborated with an experimental porosity expert to develop the model and implement it into their respective codes. This paper describes the formulation of the new boundary condition and presents an assessment of its effectiveness in simulating the aggregate aerodynamics induced by surface porosity using wind-tunnel results for a 5.0 Caliber Tangent-Ogive hody and GA(W)-1 wing model. This technique was recently utilized in a passive porosity control effector design study $[1]$ on the military aircraft configuration illustrated in Fig. 1.

\section{NOMENCLATURE}

$\begin{array}{ll}A & \text { Area } \\ a & \text { Speed of sound } \\ b & \text { Wing span } \\ C_{l,} & \text { Drag force coefficient } \\ \text { CFD } & \text { Computational Fluid Dynamics } \\ C_{l} & \text { Lift force coefficient } \\ C_{b} & \text { Pressure coefficient } \\ c & \text { Wing chord } \\ c_{b} & \text { Magnification parameter }\end{array}$




\begin{tabular}{|c|c|}
\hline$c^{\prime}=$ & Relaxation parameter \\
\hline$D$ & Model hase diameter \\
\hline$L / D$ & Lift to Drag ratio \\
\hline$M$ & Mach number \\
\hline$\dot{m}$ & Area averaged mass flux \\
\hline$N_{\text {furfons }}$ & Number of eell faces on porous boundary \\
\hline$p$ & Pressure \\
\hline$R e$ & Reynolds number \\
\hline$s$ & Solidity parameter. $A_{\text {wht }} / A_{\text {, }}$ \\
\hline$T$ & Temperature \\
\hline US & Upper surface \\
\hline$u$ & Local velocity normal to porous surface \\
\hline$x, y, z$ & Cartesian coordinates \\
\hline y & Spanwise distance \\
\hline$\alpha$ & Angle of attack (degrees) \\
\hline$\varphi$ & $\begin{array}{l}\text { Contraction coefficient, or Circumferential } \\
\text { station on slender body, } 0 \text { \& } 360 \text { deg. - } \\
\text { windward. } 180 \text { deg. - leeward }\end{array}$ \\
\hline$\gamma$ & Ratio of specific heats for air, 1.4 \\
\hline$\rho$ & Density \\
\hline
\end{tabular}

\section{Subscripts}

$\begin{array}{ll}\text { arg } & \text { averaged over surface } \\ b & \text { houndary } \\ c & \text { wing reference chord } \\ D & \text { model base reference diameter } \\ \text { domain } & \text { computational domain } \\ \text { effective } & \text { constricted orifice area } \\ \text { max } & \text { maximum } \\ \text { min } & \text { minimum } \\ \text { normal } & \text { perpendicular to porous surface } \\ n & \text { iteration number } \\ \text { porous } & \text { region of surface porosity } \\ \text { solid } & \text { solid region of porous surface } \\ t & \text { total stagnation quantity } \\ \text { tangent } & \text { tangent to porous surface } \\ \text { update } & \text { iterative update quantity } \\ \infty & \text { freestream condition } \\ l & \text { upstream flow on porous surface } \\ 2 & \text { minimum area of flow through porous surface } \\ 3 & \text { downstream. fully mixed flow through porous }\end{array}$

\section{POROUS SURFACE FLOW MODEL}

The proposed porous surface flow model builds on the general approach of Bush [22]. The Bush model was derived to pass flow information across a conterminous boundary separating an external computational grid zone from an internal plenum grid zone. The present approach is formulated as a surface boundary condition, thus eliminating the need for grid cells on the plenum side of a porous surface.

A schenatic of the porous surface model is depicted in Fig. 2 as a cut through a section of solid surface and a hole. Porosity is defined by a solidity parameter $s=A_{\text {rrit }} / A$, that quantifies the area ratio between solid and total surface. Three uniform states are assumed across the porous surface: upstream (1). minimum area (2), and downstream (fully mixed) (3). The upstream and downstream areas are equal $\left(A_{i}=A\right)$. The area at 2 is assumed to be reduced by the solidity parameter. $s$, and a contraction coefficient that represents a further area reduction due to flow through the orifice, $A_{2}=A, \varphi(/-s)$ where $\left(\varphi=A_{\text {eftithe }} / A_{f}\right)$. The contraction coefficient in Ref 122$]$ is determined from curve fits to experimental data following guidelines provided by Cornell [23] and Rouse [24]:
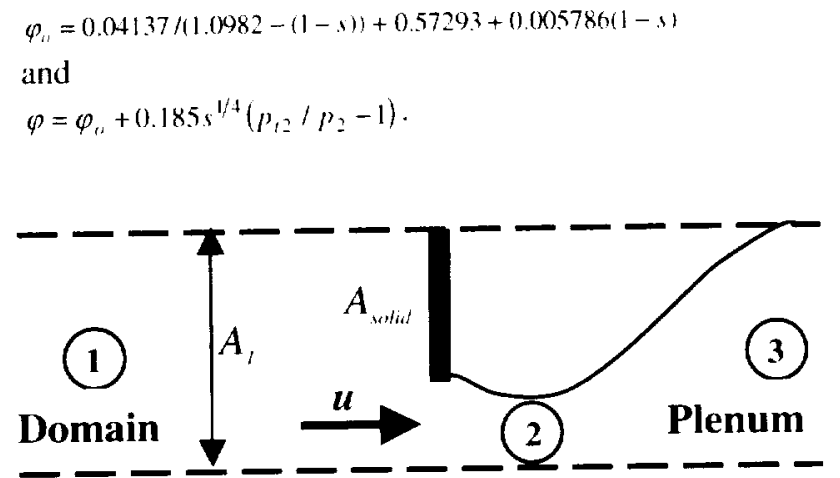

Figure 2 - Crosscut schematic of porous surface.

\section{Governing equations}

The governing equations are derived from conservation of mass and momentum (normal to the surface) for steady. one dimensional, isentropic flow of a perfect gas. Density and velocity are nondimensionalized by freestream density $\tilde{\rho}_{\infty}$ and speed of sound $\tilde{a}_{\infty}$, respectively, pressure by $\tilde{\rho}_{\infty} \tilde{a}_{\infty}^{2}$. and temperature by static freesteam temperature $\tilde{T}_{\infty}$. It is also assumed that the flow is adiabatic (i.e.. no heat (ransfer), and that there is no total pressure loss during jet formation. The latter assumption is valid as long as the fluid passing through the orifice has an inviscid core. Thus, the flow normal to the porous surface boundary is determined by the following equations.

Conservation of mass from 1-2:

$(\rho u)_{1}=(\rho u)_{2} \varphi(1-s)$ 
Conservation of mass from 1-3

$(\rho u)_{1}=(\rho u)_{3}$

No loss in jet formation from 1-2

$p_{t 1}=p_{r 2}$

Momentum halance from 2-3 with induced screen losses

$\left.p_{2} \mid 1+\not M ? \frac{2}{2} \varphi(1-s)\right]=p_{3}\left(1+\not M_{3}^{2}\right)$

Auxiliary isentropic flow relations:

$$
\begin{aligned}
& \left(\rho_{u}\right)=\frac{\gamma}{\sqrt{T_{t}}} p M\left(1+\frac{\gamma-1}{2} M^{2}\right)^{1 / 2} \\
& p_{t}=p\left(1+\frac{\gamma-1}{2} M^{2}\right)^{\gamma /(\gamma-1)}
\end{aligned}
$$

Assuming that a tangential pressure gradient exists across the surface and the net mass thux across a passively porous surface is zero, there will always be some flow transversing both into and out of the plenum at any given time. Thus. the application of the governing equations is dependent on the direction of the surface normal flow. which is a function of the local pressure gradient between the plenum and the domain. Formulations are presented below for the two conditions.

\section{Implementation}

A general assumption for the present model is that entire process from state 1 to 3 in Fig. 2 transpires over an infinitesimal distance. Hence, the application of the model is in the form of a surface boundary condition. The following will describe the proposed boundary condition for flow moving both into $\left(p_{\text {itemam }}<\right.$ $\left.p_{\text {thmanum }}\right)$ and out of $\left(p_{\text {menum }}>p_{\text {dumitum }}\right)$ the plenum. The local velocity, $t$, is defined as the component normal to the surface.

Flow into plenum $\left(p_{\text {m:num: }}<p_{\text {ditmutio }}\right)$ :

The configuration for flow into the plenum from the domain is presented in Fig. 2. The first step is to assume choked flow by assuming $M_{2}^{2}=1$ and solve Eq. 1 using Eq. 5 written at state 3, and Eq. 4 for $M_{3}^{2}$ via Newton iteration

$$
\frac{\gamma+1}{2}\left[\frac{1+M_{3}^{2}}{1+\gamma \varphi(1-s)}\right]^{2} \varphi^{2}(1-s)^{2}-M_{2}^{2}\left(1+\frac{\gamma-1}{2} M_{3}^{2}\right)=0
$$

From this result compute the maximum mass flow using Eq. 5 written at state 3

$$
\left(\rho_{u}\right)_{\max }=\frac{\gamma}{\sqrt{T_{i}}} p_{3} M_{3}\left(1+\frac{\gamma-1}{2} M_{3}^{2}\right)^{1 / 2}
$$

If $(\rho u)_{1} \leq(\rho u)_{\max }$, i.e. not choked, then solve Eq. 2 using Eq. 5 written at state 3 for $M_{3}^{2}$ :

$$
M_{3}^{2}=\frac{-1+\sqrt{1+2\left((\gamma-1) / \gamma^{2}\right) T_{t}\left(\rho_{H}\right)_{1}^{2} / p_{3}^{2}}}{\gamma-1}
$$

then solve Eq. I using Eq. 5 written at state 2. and Eq. 4 for $M_{2}^{2}$ via Newton iteration:

$$
\begin{aligned}
& M_{2}^{2}\left(1+\frac{\gamma-1}{2} M_{2}^{2}\left[\frac{1+M_{3}^{2}}{1+M_{2}^{2} \varphi(1-s)}\right]^{2} \varphi^{2}(1-s)^{2}\right. \\
& -\frac{T_{1}}{\gamma^{2}} \frac{(\rho u)_{1}^{2}}{p_{3}^{2}}=0
\end{aligned}
$$

If flow is choked, $(\rho u)_{1}>(\rho u)_{\max }$, then set $(\rho u)_{1}=(\rho u)_{\max }$ and $M_{2}^{2}=1$. Now use Eqs. 3 and 4 with 6 written at state 2 to compute $p_{t l}$ :

$$
p_{11}=p_{3} \frac{1+\not M_{3}^{2}}{1+\not M_{2}^{2} \varphi(1-s)}\left(1+\frac{\gamma-1}{2} M_{2}^{2}\right)^{\gamma /(\gamma-1)}
$$

then compute $M_{1}^{2}$ using Eq. 5 written at state I via. Newton iteration:

$M_{1}^{2}\left(1+\frac{\gamma-1}{2} M_{1}^{2}\right)^{-\left(\frac{\gamma+1}{\gamma-1}\right)}-\frac{T_{t}}{\gamma^{2}} \frac{(\rho u)_{1}^{2}}{p_{t 1}^{2}}=0$

We now have sufficient information to compute the boundary flux using $\left(\rho_{u}\right)_{1}, M_{1}^{2}$ and $p_{t \prime}$.

$$
\begin{aligned}
& p_{b}=p_{t 1}\left(1+\frac{\gamma-1}{2} M_{1}^{2}\right)^{-\left(\frac{\gamma}{\gamma-1}\right)} \\
& \rho_{b}=\frac{p_{b}}{T_{i}}\left(1+\frac{\gamma-1}{2} M_{1}^{2}\right) \\
& u_{b \text {, nurmul }}=(\rho u)_{1} / \rho_{h}
\end{aligned}
$$

$u_{\text {1, tratsent }}$ is zero for a viscous boundary or the inviscid tangential velocity for an inviscid boundary. 


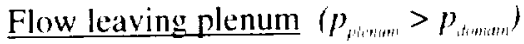

The configuration for flow into the domain from the plenum is presented in Fig. 3. The first step is 10 once again check for choked flow by assuming $M_{2}^{2}=1$ and solving Eq. 1 using Eq. 5 written at state 1, and Eq. 3 for $M_{\Gamma}^{2}$ via Newton iteration:

$\left[\left(\frac{2}{\gamma+1}\right)\left(1+\frac{\gamma-1}{2} M_{1}^{2}\right)\right]^{\frac{\gamma+1}{\gamma-1}} \varphi^{2}(1-s)^{2}-M_{1}^{2}=0$

From this result compute the maximum mass flow using Eq. 5 written at statc 1:

$(\rho i 1)_{\max }=\frac{\gamma}{\sqrt{T_{1}}} p_{1} M_{1}\left(1+\frac{\gamma-1}{2} M_{1}^{2}\right)^{1 / 2}$

If $(\rho u)_{3} \leq(\rho u)_{\max }$. i.e. not choked. then solve Eq. 2 using Eq. 5 written at state 1 for $M_{1}^{2}$ :

$M_{1}^{2}=\frac{-1+\sqrt{1+2 \frac{\gamma-1}{\gamma^{2}} T_{t} \frac{(\rho u)_{3}^{2}}{p_{1}^{2}}}}{\gamma-1}$

then solve Eq. 1 using Eq. 5 written at state 2. and Eq. 3 for $M_{2}^{2}$ via Newton iteration:

$$
\begin{aligned}
& \left(1+\frac{\gamma-1}{2} M_{1}^{2}\right)^{\frac{2 \gamma}{\gamma-1}} M_{2}^{2}\left(1+\frac{\gamma-1}{2} M_{2}^{2}\right)^{-\left(\frac{\gamma+1}{\gamma-1}\right)} \varphi^{2}(1-s)^{2} \\
& -\frac{T_{1}}{\gamma^{2}} \frac{\left(\rho_{l}\right)_{3}^{2}}{p_{1}^{2}}=0
\end{aligned}
$$

If flow is choked, $(\rho u)_{3}>(\rho u)_{\max }$, then set $(\rho u)_{3}=(\rho u)_{\max }$ and $M_{2}^{2}=1$. Now Eqs. 3 and 6 to compute pressure at state 2 :

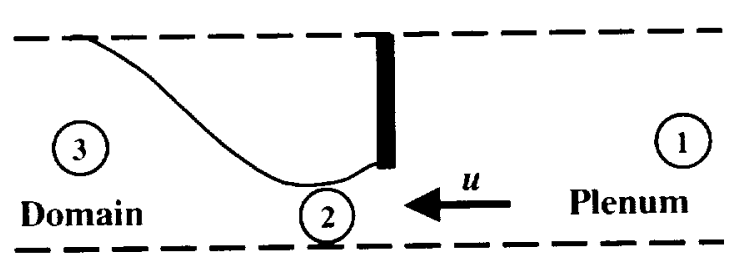

Figure 3 - Model for flow leaving plenum.

$$
p_{2}=p_{1}\left(\frac{1+\frac{\gamma-1}{2} M_{1}^{2}}{1+\frac{\gamma-1}{2} M_{2}^{2}}\right)^{\gamma /(\gamma-1)}
$$

then solve Eq. 2 using Eq. 5 written at state 3 for $M_{3}$.

$$
\begin{aligned}
& M_{3}^{2}\left(1+\frac{\gamma-1}{2} M_{3}^{2}\left[\frac{1+\gamma M_{2}^{2} \varphi(1-s)}{1+M_{3}^{2}}\right]^{2}\right. \\
& -\frac{T_{t}}{\gamma^{2}} \frac{(\rho u)_{3}^{2}}{p_{2}^{2}}=0
\end{aligned}
$$

Use Eq. 6 written at stute 3 and Eq. 4 to compule $p_{t 3}$ :

$$
p_{t 3}=p_{2} \frac{1+M_{2}^{2} \varphi(1-s)}{1+\gamma M_{3}^{2}}\left(1+\frac{\gamma-1}{2} M_{3}^{2}\right)^{\frac{\gamma}{\gamma-1}}
$$

There is now sufficient information to compute the boundary flux using $(\rho u)_{3}, M_{3}^{2}$ and $p_{t 3}$.

$$
\begin{aligned}
& p_{b}=p_{13}\left(1+\frac{\gamma-1}{2} M_{3}^{2}\right)^{\left(\frac{\gamma}{\gamma-1}\right)} \\
& \rho_{h}=\frac{p_{n}}{T_{1}}\left(1+\frac{\gamma-1}{2} M_{3}^{2}\right) \\
& u_{h, n, \ldots, m u l l}=(\rho u)_{3} / \rho_{l}
\end{aligned}
$$

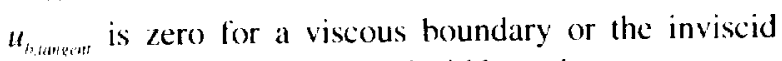
tangential velocity for an inviscid boundary.

\section{Determination of Plenum Pressure}

A plenum pressure must be determined which yields a zero net mass flux balance across the porous surface. This pressure is used for $p_{3}$ in Eqns.

8-11, and for $p_{1}$ in Eqns. 14-17. The following describes an iterative procedure for estimating plenum pressure.

First compute an area-averaged pressure and normal mass flux over the porous surface.

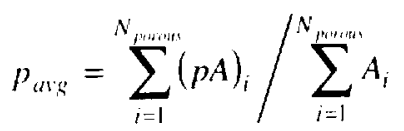

\footnotetext{
' One could conceivably prescribe a non-zero net mass flux to simulate blowing or suction. This has not been tested in present work.
} 


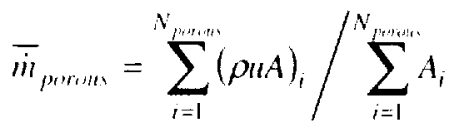

Assume the initial value of plenum pressure to be the averaged pressure $\left(p_{\text {plemum. }}=p_{\text {ane }}\right)$.

On the second iteration. compute a bracketed update (o) plenum pressure:

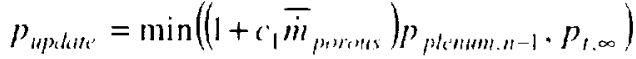

$$
\begin{aligned}
& p_{\text {upridut }}=\max \left(p_{\text {updete }}, 1.005 p_{\text {min }}\right)
\end{aligned}
$$

where $c_{1}=10$ is a magnilication parameter. $p_{t, \infty}$ the freestream total pressure, and $p_{\min }$ the minimum value of pressure on porous surface from summation for $p_{x a y}$.

It is necessary to impose a filter on the pressure updates to damp the temporal oscillations.

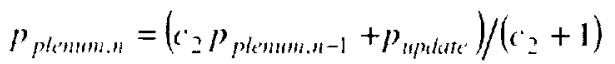

where the relaxation parameter $c_{2}=50$.

\section{DESCRIPTION OF FLOW SOLVERS}

The porous boundary condition has been implemented in two structured-grid flow solvers. TLNS3D and CFL.3D, and one unstructured solver. USM3Dns. A brief description of the salient features of each code is included below.

\section{TLNS3D}

The TLNS3D (Thin Layer Navier-Stokes Solver for 3-D flows) code [25.26] uses a cellcentered. finite-volume approach for solving inviscid and viscous flows over complex configurations on general multi-block structured grids. A suite of algebraic and one- and two-equation turbulence models is used for simulating turbulent flows. Artificial dissipation is added to the central-difference scheme for stability. TLNS3D also makes use of local time stepping, implicit residual smoothing and multigrid techniques in conjunction with multi-stage Runge-Kutta time-stepping scheme to accelerate the convergence of the code to steady-state solutions.

\section{CFL3D}

CFL3D [27] solves the three-dimensional. time-dependent. thin-layer approximation to the
Reynolds-Averaged Navier-Stokes (RANS) equations using a finite volume formulation in generalized coordinates. It uses upwind-biased spatial differencing with Roe's [28] flux-difference splitting or Van Leer's [29] flux-vector splitting methods for the inviscid terms, and central differences for the viscous and heat transfer terms. The code, which is second-order accurate in space. is advanced in tine with an implicit three-factor approximate factorization (AF) scheme. Temporal subiterations with multigrid are used 10 recover time accuracy lost as a result of the AF approach during unsteady calculations. The code includes several grid connection strategies. a vast array of zero-, one- and two-equation turbulence models (linear as well as nonlinear), and numerous boundary conditions. The results presented in this paper were obtained using the two-equation $k-\omega$ SST model of Menter |30|.

\section{USM3Dns}

USM3Dns [3I] is a tetrahedral cell-centered. finite volume Euler and Navier-Stokes flow solver. Inviscid flux quantities are computed across each cell face using Roe's [28] flux-difference splitting. Spatial discretization is accomplished by a novel reconstruction process that is based on an analytical formulation for computing solution gradients within tetrahedral cells. The solution is advanced to a steady state condition by an implicit backward-Euler timestepping scheme. Flow turbulence effects are modeled by the Spalart-Allmaras one-equation turbulence model [32]. The model can be integrated all the way to the wall, or can be coupled with a wall function to reduce the number of cells in the sublayer

\section{RESULTS AND DISCUSSION}

An assessment of the new porous boundary condition is made with two selected examples using the three flow solvers. The first is a 5.0 caliber tangent-ogive with and without forebody porosity. Without porosity. the forehody develops strong vortex asymmetries at high angles of attack that generate severe yawing moments. Surface porosity alleviates these asymmetries.

The second case is the General Aviation (Whitcomb) - I wing (GA(W)-1) with and without leading-edge porosity. The effect of the porosity is to reduce lift and increase drag. Such porosity is conceived as a conformal control device when applied asymmetrically 10 a wing 10 generate rolling and yawing moments. 


\subsection{Caliber Tangent-Ogive Forebody}

A 5.0 caliber tangent-ogive forebody was tested, as one of a family of ogives, in the Langley $7 \times 10$ Foot High-Speed Wind Tunnel to investigate the effect of fineness ratio on the asymmetric loading of slender forebodies and the effectiveness of passive porosity in alleviating these asymmetries [10]. Figure 4 presents a full surface representation of the forebody. The original sting-mounted wind-tunnel model is 30 inches long and 4 inches diameter. For the computational geometry depicted in Figure 4. the sting has been removed and the body extended to 40 inches in length where it is terminated at an outflow boundary. Surface porosity is applied to the darkened region which extends from $x=1$ to 20 inches.

The computation of asymmetric vortex flows on slender ogive hodies at high angles of attack presents a challenging problem [33.34] that is beyond the scope of this paper. Such flows are characterized by massive crossflow separation with asymmetric feedback through the boundary layer, and are highly sensitive to laminar-to-turbulent transition location. turbulence models, and numerical discretization. The present work is focused on the formulation and verification of the porous boundary condition. Since surface porosity restores flow symmetry, most of the following assessments will be performed on symmetric half-plane grids. One full-body asymmetric flow solution will be included for completeness.

Both structured-hexahedral and unstructured tetrahedral grids were constructed for the study. The structured grid for TLNS3D and CFL3D contained $177 \times 97 \times 65$ cells, and the unstructured grid for USM3Dns had 1,009,929 cells. The USM3Dns grid is mirrored for the full-body computation. Fartield boundaries were placed at $\mathrm{x}_{m+1,}, \mathrm{y}_{\text {nim }}$. and $z_{m, i}$ of $-120,0$.

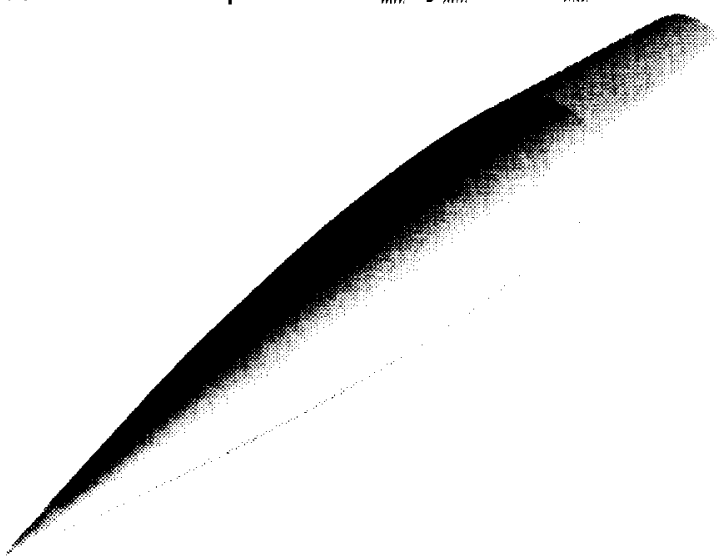

Figure 4 - Surface representation of 5.0 caliber tangent ogive configuration. Porous region denoted by shading. and -150 inches, and $x_{\text {mix }}, y_{\text {mat }}$, and $z_{\text {mat }}$ of 40.180 . and 150 inches, respectively. A characteristic inflow/outflow boundary condition was applied to the inflow, top and side boundaries. An outflow extrapolation condition was prescribed to the aft boundary. The no-slip condition was applied to the solid surfaces on the ogive. with the exception that USM3Dns utilized a wall function. A 22-percent porosity $(s=0.78)$ boundary condition is applied to the darkened forebody surface region denoted in Fig. 4 .

Navier-Stokes solutions were computed on the ogive body with the three flow codes at $M_{\alpha}=0.3$. $\alpha=30$ and 40 degrees, and $R e_{1}=0.4$ million. TLNS3D and USM3Dns utilized the Spalart-Allmaras oneequation urbulence model and CFL.3D the Menter SST two-equation model. The solid solutions were computed first. Then the porous boundary condition was activated and the solutions restarted. Figure 5 presents the typical convergence of the surface net mass flux, and the plenum and surface averaged pressures for the porous region after restarting from a converged solid surface solution. Note that the plenum pressure is substantially different than the surface-averaged value. Earlier studies assessed the use of surface-averaged pressure as the plenum pressure with the porosity boundary condition. This approach was determined to yield inaccurate results. which led to the development of the iterative procedure for plenum pressure described earlier.

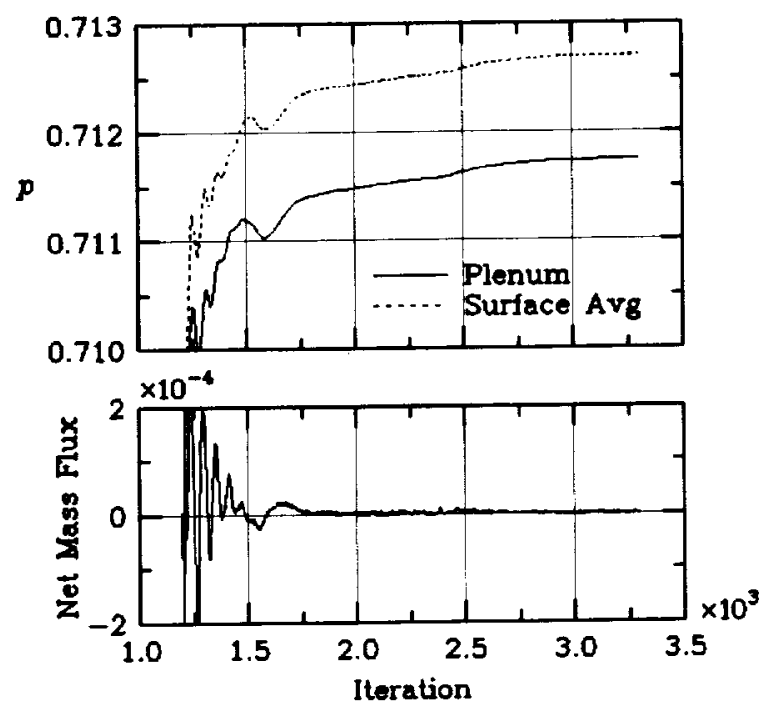

Figure 5 - Convergence of the net mass flux, and the plenum and surface averaged pressures over the porous region of 5.0-caliber tangent-ogive forebody from USM3Dns; $M_{\infty}=0.3, \alpha=30 \mathrm{deg}$, and $\mathbf{R e}_{11}=\mathbf{0 . 4}$ million. 
Implementation of this procedure was only completed for USM3Dns in time for publication. For the results presented herein. the values for plenum pressure were determined from the USM3Dns solutions and provided as inpul for the other codes. The final nondimensional plenum pressures for $\alpha=30$ and 40 degrees were 0.7118 and 0.7099 , respectively, where nondimensional free-stream pressure is 0.7143 . Nondimensionalization is discussed in earlier section on Governing Equations.

The effect of porosity on the offbody flow field is evident in Fig. 6. Contours of density are shown at station $x=10$ inches $(x / D=2.5)$ for $\alpha=30 \mathrm{deg}$ for the solid-surlace solution (left) and porous-surface solution (right). The tightly clustered contour lines over the solid surface denote the presence of a strong vortex core, whereas the lines over the porous surface suggest a more diffused vortical system.

A comparison of surface circumferential $C$ distributions is shown in Figs. 7 and 8 at $x=10$ inches $(x / D)=2.5)$ for angles of attack 30- and 40-degrees. respectively. A strong asymmetry is evident in the experimental distributions for the solid surface. which is the source of unwanted load asymmetries on the ogive. The beneficial effect of passive porosity is evidenced in the companion data by a diffusion of the vortex suction peaks into a symmetric distribution.

A full-grid solution from USM3Dns is included in Figs. 7 and 8 to illustrate the difficulty of computing accurate surface pressure distributions with a solid (non-porous) surface for this class of problem. Note that asymmetric solutions are produced. but accuracy is marginal. These solutions were

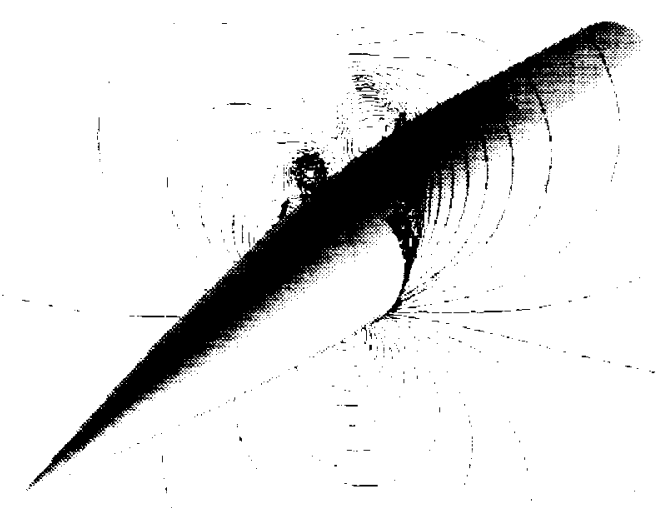

Figure 6. - Contours of density at $\mathrm{x} / \mathrm{D}=2.5$. (Solid surface - left; porous surface -- right.) 5.0 caliber tangent-ogive forebody from USM3Dns; $M_{\infty}=0.3$, $\alpha=30 \mathrm{deg}$, and $\mathrm{Re}_{\mathrm{t}}=\mathbf{0 . 4}$ million. generated at zero sideslip with an initial asymmetry triggered by applying asymmetric viscous boundary conditions on the nose ahead of $x=1$; a wall function on the starboard and a no-slip condition on the port. Subsequent porous computations (not shown) were

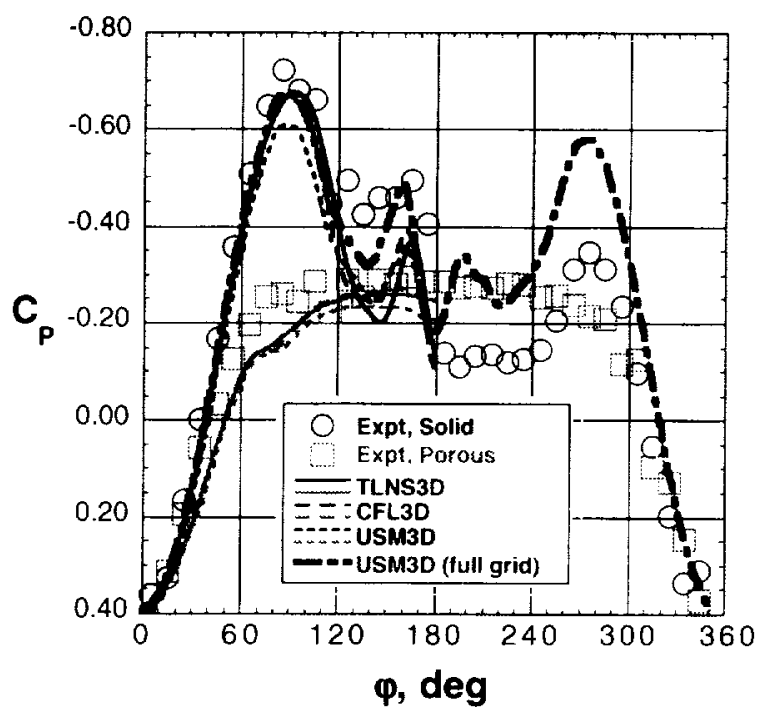

Figure 7 - Code to code comparison of circumferential $C_{p}$ distribution on 5.0 caliber tangent-ogive forebody with and without porosity. Station $x / D=2.5, M_{\infty}=0.3, \alpha=30 \mathrm{deg}$, and $R_{1}=0.4$ million.

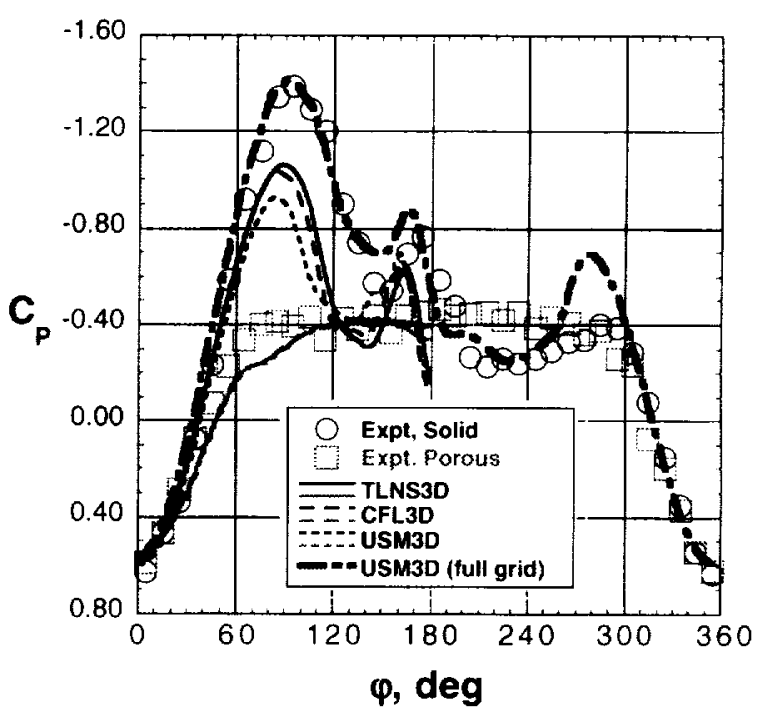

Figure 8 - Code to code comparison of circumferential $C_{p}$ distribution on 5.0 caliber tangent ogive forebody with and without porosity. Station $x / D=2.5, M_{\infty}=0.3, \alpha=40 \mathrm{deg}$, and $\operatorname{Re}_{11}=0.4$ million. 
restarted from the asymmetric solutions. Flow symmetry was restored even wirh the asymmetric viscous boundary conditions set ahead of $x=1$.

The computational $C_{b}$, distributions on the half-plane grids are presented on Figs. 7 and 8 between $\varphi=0$ and 180 degrees The distributions from the solid (non-porous) solutions are included to facilitate codeto-code comparisons. and are not intended to reflect a correct solution to an inherently asymmetric problem. Some differences in $C_{n}$ distribution are noted between codes. but each qualitatively captures the dominant vortex flow fealures. The focus is on the porous surface results which demonstrate good quantitative agreement with the experimental data. This confirms the adequacy of the new porous surface boundary condition for computing aggregate aerodynamic effects of passive porosity for this class of problem.

\section{GA(W)-I Wing}

The GA(W)- I [General Aviation (Whitcomb) - 11 wing was tested in the 14- by 22-Foot Subsonic Wind Tunnel. This model had a 9-foot span, 3-foot chord. and $0^{\circ}$ leading-edge sweep. Surface pressure taps were located at 3 spanwise locations. The model was also equipped with porous skins to represent a passive porous test article (porous surface with plenum cavity)

The semispan surface definition used for the computations is shown in Fig. 9. Porosity was applied to the shaded leading-edge region ahead of the 18 pereent chord station. Computational grids were constructed for each of the flow solvers. The grid for TLNS3D an CFL3D contained $193 \times 65 \times 3.3$ hexahedral cells, whereas for USM3Dns the grid contained $1.681,831$ tetrahedral cells. Farfield boundaries were placed 10 chord lengths away from the wing in all directions on which a characteristic inflow/outflow boundary condition was applied. The no-slip condition was applied to the wing solid surfaces, with the exception that USM3Dns utilized a wall function. When applying leading-edge porosity, a 22-percent condition $(s=0.78)$ was prescribed to the darkened region denoted in Fig. 9.

Navier-Stokes flow solutions were computed at $M_{\infty}=0.2, \alpha=0$ and 8 degrees, and a chord Reynolds number of 3.5 million. As before, the plenum pressure was determined from USM3Dns and provided as input for the other codes. The nondimensional plenum values used for $\alpha=0$ and 8 degrees were 0.7130 and 0.7156. respectively.

Figures 10 compares the chordwise $C p$ distributions at $\alpha=8$ deg. and $2 y / h=0.67$ (one chord length from the symmetry plane) between the code results and experimental data for the solid and porous surfaces. The experimental data reveals a dramatic loss of leading-edge suction peak and consequent loss of lift due to passive porosity. The solid surface computational results are nearly identical between the codes and are in generally good agreement with the experimental data. with the exception of the leadingedge suction peak. The porous leading-edge computations show some variation between codes, but are in reasonably good agreement with the data.

Figures 11 and 12 illustrate the large impact of leading-edge porosity on lift and drag coefficients.

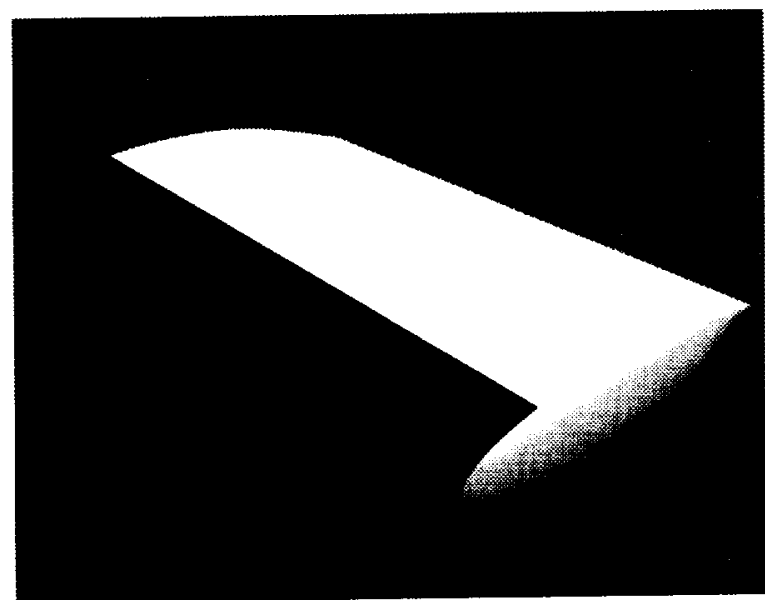

Figure 9 - Semispan surface geometry for $\mathrm{GA}(\mathrm{W})-1$ wing. Porosity applied to shaded region around leading edge

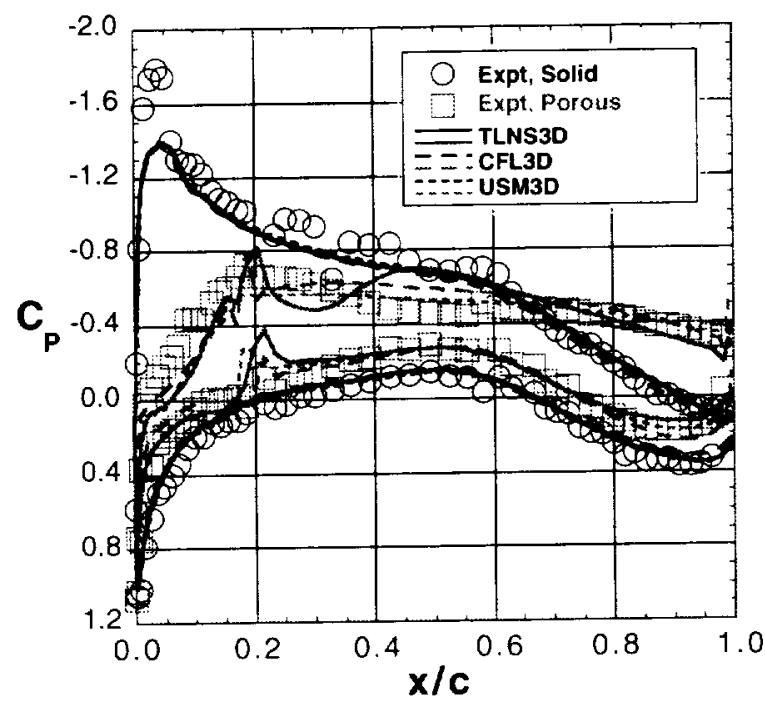

Figure 10 - Cp comparison of solid and porous GA(W)-1 wing for TLNS3D and USM3Dns with experiment at $2 \mathrm{y} / \mathrm{b}=0.67 . \mathrm{M}_{\infty}=0.17, \alpha=8 \mathrm{deg}$., and $\operatorname{Re}_{c}=3.5$ million 
and demonstrate that the porosity boundary condition model yields correct estimates of those effects at angles of attack of 0 and 8 degrees. With leading-edge porosity having such a large effect on lift and drag, an asymmetric application of this device could be envisioned for lateral-directional control in place of moving control surfaces. The computational model presented herein should be useful as a supplemental design tool in what was previously an experimental intensive process.

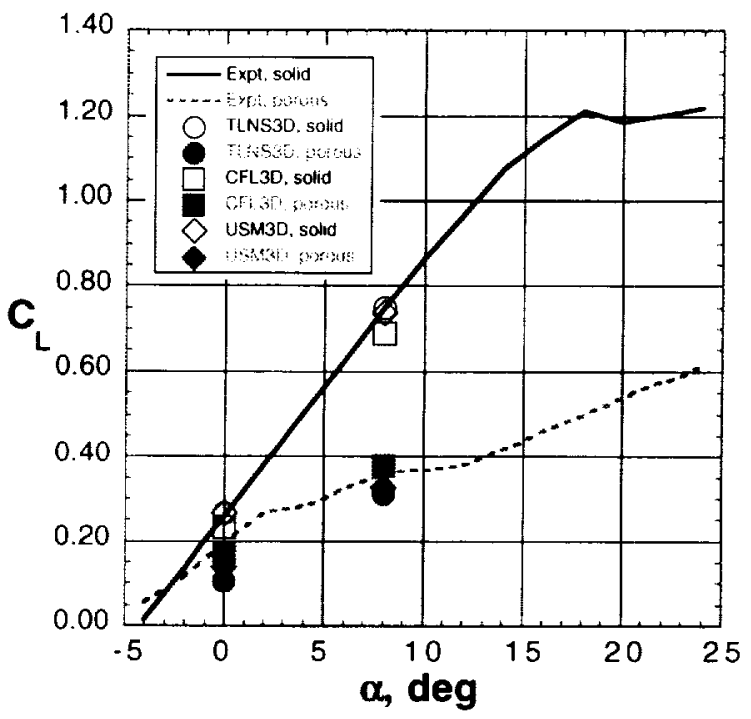

Figure 11 - Comparison of lift characteristics of solid and porous leading edge for $G A(W)-1$ wing. $M_{\infty}=0.17$ and $\operatorname{Re}_{\mathrm{s}}=\mathbf{3 . 5}$ million.

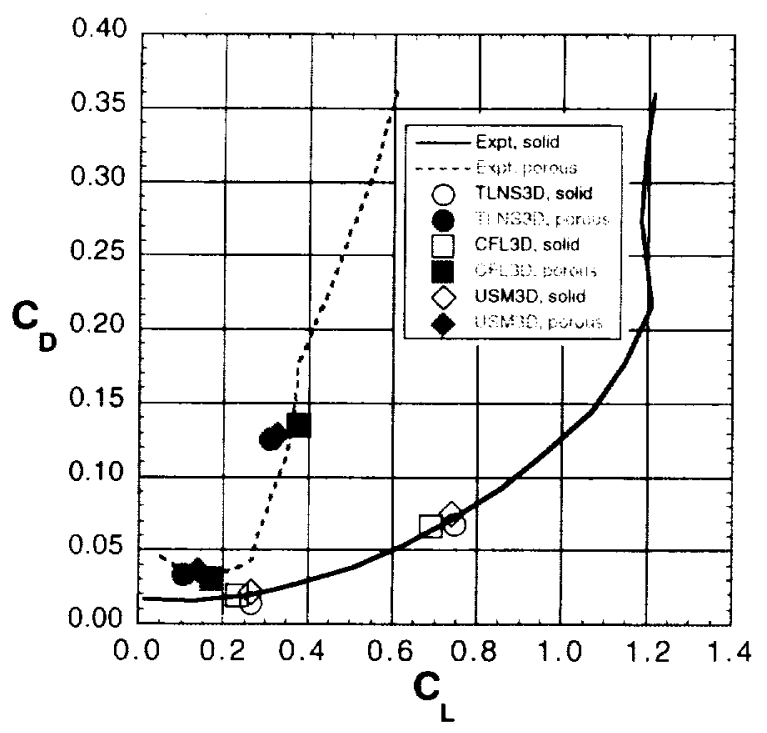

Figure 12 - Comparison of drag characteristics of solid and porous leading edge for $\mathrm{GA}(W)-1$ wing. $M_{\infty}=0.17$ and $\operatorname{Re}_{i}=\mathbf{3 . 5}$ million.

\section{CONCLUSIONS}

A new boundary condition is presented for simulating the flow over porous surlaces. The model builds on the prior work of Bush to eliminate the need for constructing grid within an underlying plenum. thereby simplifying the numerical modeling of passively porous flow control systems and reducing computation cost.

Code experts for two structured-grid flow solvers. TLNS3D and CFL.3D, and one unstructured solver, USM3Dns, collaborated with an experimental porosity expert to develop the model and implement it into their respective codes. Results presented for the three codes on a slender forebody with porosity, and a wing with leading-edge porosity demonstrate good agreement with experimental data and a remarkable ability to predict the aggregate aerodynamic effects of surface porosity with a simple boundary condition.

Experimental studies of surface porosity have shown the strong potential for this technology as a flow control device. Porosity has many potential applications for aerodynamic control. drag reduction/production, separation control, and lift improvement. The present work should facilitate a more complete understanding of surface porosity in the future by enabling complementary computational studies and more timely design trades.

\section{REFERENCES}

1. Hunter, C. A.. Viken, S. A., Wood, R. M., and Bauer. S. X. S.: Advanced Aerodynamic Design of Passive Porosity Control Effectors. 39" AIAA Acrospace Sciences Meeting and Exhibit, 8-11 January 2001, Reno, NV. AIAA 2001-0249.

2. Thiede, P.. Krogmann. P.. and Stanewsky. E.. "Active and Passive Shock/Boundary Layer Interaction Control On Supercritical Airfoils." AGARD-CP-365, May 1984.

3. Bahi, L. and Ross. J. M., "Passive Shock Wave/Boundary Layer Control for Transonic Airfoil Drag Reduction." AIAA-83-(0137, January 1983.

4. Nagamatsu, H. T., Brower, W. B., Jr., Bahi, L.; and Marble, S. K.. "Investigation of Passive Shock Wave/Boundary Layer Control for Transonic Airfoil Drag Reduction." Rensselear Polytechnic Institute, Troy, NY, First Annual Report for NASA Grant No. NSG 1624, October 1. 1979 to September 30. 1980. 
5. Savu, G., Trifu, O., and Dumitrescu, L. Z., "Suppression of Shocks on Transonic Airfoils," Proceedings of the International Symposium on Shock Tubes and Waves. Sydney. August 19-22. 1983.

6. Savu, G. and Trifu, O., "Porous Airfoils in Transonic Flow," AIAA Journal, Vol. 22, No. 7. July 1984, pages 989-991.

7. Nagamatsu, H. T. and Orozco, R. D.. "Porosity Effect on Supercritical Airfoil Drag Reduction by Shock Wave/Boundary Layer Control." AIAA-841682, June 1984

8. Bauer, S. X. S. and Hernandez. G.. "Reduction of Cross-Flow Shock-Induced Separation with a Porous Cavity al Supersonic Speeds." AIAA-882567. June 1988.

9. Wood, R. M.; Banks. D. W.: and Bauer. S. X. S.: Assessment of Passive Porosity with Free And Fixed Separation on a Tangent-Ogive Forebody. Presented at the AIAA Atmospheric Flight Mechanics Conference. Hilton Head, South Carolina, Augusi 10-12, 1992. AIAA Paper No. 92-4494.

10. Bauer. S. X. S. and Hemsch. M. J.: Alleviation of Side Foree on Tangent-Ogive Forebodies Using Passive Porosity, Journal of Aircraft. Volume 31. Number 2. Pages 354-361. 1993.

11. Allen, H. J. and Perkins, E. W.. "A Study of the Effects of Viscosity on Flow Over Slender Inclined bodies of Revolution," NACA TR 1048, December 1951.

12. Letko, W.. "A Low-Speed Experimental Study of the Directional Characteristics of a Sharp-Nosed Fuselage Through a Large Angle-of-Atack Range at Zero Angle of Sideslip." NACA TN 2911, July 1953.

13. Coe, P. L., Jr.. Chambers, J. R., and Letko, W., "Asymmetric Lateral-Directional Characteristics of Pointed Bodies of Revolution at High Angles of Attack," NASA TN D-7095, November 1972.

14. Pick, G. S., "Investigation of Side Forces on Ogive-Cylinder Bodies at High Angles of Altack in the $\mathrm{M}=0.5$ to 1.1 Range," AlAA Paper 71-570, June 1971

15. Clark, W. H. and Peoples, J. R., "Occurrence and Inhibition of Large Yawing Moments During High Incidence Flight of Slender Missile Configurations," AIAA Paper 72-968, Sept. 1972.

16. Jorgensen, L. H. and Nelson, E. R., "Experimental Acrodynamic Characteristics for a Cylindrical Body of Revolution With Various Noses at Angles of Altack from $0^{\circ}$ to $58^{\circ}$ and Mach Numbers from 0.6 to 2.0." NASA TM X-3128. December 1974.
17. Jorgensen, L. H. and Nelson, E. R., "Experimental Aerodynamic Characteristics for a Cylindrical Body of Revolution With Side Strakes and Various Noses at Angles of Allack from $0^{\circ}$ to 58 and Mach Numbers from 0.6 to 2.0." NASA TM X-3130, March 1975 .

18. Jorgensen, L. H. and Nelson, E. R., "Experimental Aerodynamic Characteristics for Bodies of Elliptic Cross Section at Angles of Altack from $0^{\circ}$ to $58^{\circ}$ and Mach Numbers from 0.6 to 2.0." NASA TM X-3129. February 1975.

19. Morgan. H. L.. Jr. and Pautson. J. W.. Jr.: Acrodynamic characteristics of wing-body configuration with two advanced general aviation airfoil sections and simple flap systems. NASATN-D-8524. Aug (01, 1977.

20. Hartwich. P. M.: Euler study on porous transonic airfoils with a view loward multipoint design. Journal of Aircraft. Volume: 30 Issue: 2 Page: p. 184-191. Apr ()1. 1993.

21. Kraushaar. S. L. and Chokani. N.: Improved Models for the Porous Surface with Passive Control, Masters Thesis North Carolina State University. Jan 01. 1996.

22. Bush. R. H.: "Engine Face and Screen Loss Models for CFD Applications." AIAA Paper 972076. June 1997.

23. Cornell, W. G.: "Losses in Flow Normal to Plane Screens," Transactions of the ASME. pp 791-799. May 1958.

24. Rouse, H.. "Elementary Mechanics of Fluids," Dover Publications, 1978 (originally published John Wiley and Sons. Inc.. 1946). p57.

25. Vatsa. V.N. and Wedan. B.W.: "Development of a Multigrid Code for 3-D Navier-Stokes Equations and its application to a grid-refinement Study." Computers and Fluids, Vol. 18, No. 4, pp 391403. 1990.

26. Vatsa, V.N., Sanetrik, M.D. and Parlette, E.B. "Development of a llexible and efficient multigrid-based multiblock flow solver." AIAA Paper No. 93-0677. Jan. 1993

27. Krist. S. L., Biedron. R. T., and Rumsey. C. L. "CFL3D User"s Manual". November 1996.

28. Roe, P.L., "Approximate Ricmann Solvers. Parameter Vectors, and Difference Schemes." J Comp. Phys., Vol. 43. No. 2, 1981, pp. 357-372.

29. Van Leer, B., "Flux Vector Splitting for the Euler Equations," Lecture Notes in Physics. Vol. 170. 1982. pp. 501-512.

30. Menter. F.: "Zonal Two Equation k- $\omega$ Turbulence Models for Acrodynamic Flows". AIAA 93-2906. 1996. 
31. Frink N. "Tetrahedral Unstructured Navier-Stokes Method for Turbulent Flows." AIAA Journal. Vol. 36. No. 11, pp 1975-1982, November 1998.

32. Spalart. P.R.. and Allmaras, S.R., "A OneEquation Turbulence Model for Aerodynamic Flows," AIAA Paper No. 92-0439. 1992.

3.3. Hartwich. P.M.. and Hall. R. M. "Navier-Stokes Solutions for Vortical Flows over a Tangent Ogive
Cylinder." AlAA Journal, Vol. 28. No. 7. pp 1171 1179. July 1990.

34. Hartwich. P.M.. Hall. R. M., and Hemsch. M.J. "Navier-Stokes Computations of Vortex Asymmetries Controlled by Small Surface Imperfections." J. of Spacecraft and Rockets, Vol. 28. No. 2. pp 258-264. March-April 1991. 
. 